\title{
Evaluating the Effect of Ad Hoc Routing on TCP Performance in IEEE 802.11 Based MANETs
}

\author{
Evgeny Osipov ${ }^{1, \star}$ and Christian Tschudin ${ }^{2}$ \\ 1 RWTH Aachen University, Department of Wireless Networks, Kackertstrasse 9, \\ D-52072 Aachen, Germany \\ 2 University of Basel, Computer Science Department, Bernoullistrasse 16, CH-4056 \\ Basel, Switzerland
}

\begin{abstract}
In this paper we analyze the impact of different ad hoc routing schemes on TCP traffic in multihop ad hoc wireless networks. Our hypothesis is that beyond a certain network size the uncontrolled broadcast transmission of routing messages will seriously degrade the performance of the TCP protocol. We experimentally determine the admissible operation ranges of MANETs where the level of such degradation is still acceptable for end users.
\end{abstract}

\section{Introduction}

The motivation behind studying the effect of routing traffic on TCP performance in multihop wireless ad hoc networks is twofold. On one hand, the research on benchmarking different ad hoc routing protocols is mainly conducted using UDP based CBR traffic wherefore, there is no established methodology for the analysis of TCP+routing interactions in MANETs. On the other hand, the TCP protocol in IEEE 802.11 based MANETs performs poorly even without routing and it is interesting to explore to which extend the routing traffic exacerbates the situation. Generally speaking the second problem is a direct reason for the first one. It is the unstable and unpredictable performance of TCP in MANETs which motivated the choice of CBR traffic in pioneering (e.g. [1]) and subsequent studies of ad hoc routing protocols.

In 2 the authors show that multiple TCP sessions suffer from a severe unfairness in multihop wireless networks with static routing and no mobility; At the end some sessions gain full access to the network capacity completely shutting down the unlucky competitors. The problem is linked to the inability of TCP's congestion control to differentiate the packets losses due to radio interferences from those induced by network congestion [3, 4, 5]. Obviously, the traffic generated by a routing protocol increases the contention at the physical layer and will cause packet losses in addition to those caused by radio collisions between the TCP segments themselves.

* A significant part of this work has been performed in the University of Basel while the first author was working there. 


\subsection{Contribution of the Paper}

In this paper we consider reactive ad hoc routing schemes operating in ad hoc networks with potentially perfect connectivity and no mobility. With such settings we are able to isolate the traffic generated by the routing protocol during the path maintenance phase. Based on the AODV [ 6] and LUNAR [7] on-demand ad hoc routing protocols we study the effect of four distinct traffic patterns on the quality of ongoing TCP sessions.

For each considered routing scheme we determine the network configurations in terms of the number of sustained TCP sessions and the number of nodes in the network where the ad hoc routing does not seriously affect the quality of TCP communications. We call the set of such network configurations the "admissible operation range" (AOR) for the particular routing protocol. There is a limit of AOR, which we call the "routing ad hoc horizon", beyond which the uncontrolled broadcast transmission of routing messages results in a serious degradation of TCP performance. We estimate the "Equivalent routing load" which indicates the level of this degradation.

\subsection{Outline}

We develop the topic as follows. We first review the existing experimental studies of interactions between ad hoc routing and TCP in Sect. 2, After that in Sect. 3 we introduce the problem of evaluating the effect of routing on TCP performance and outline our methodology. Section 4 is the main section where we develop our approach to analyze TCP+routing interactions in IEEE 802.11 based ad hoc networks. We summarize the material and conclude the paper in Sect. 5.

\section{Related Work}

We found very few papers which investigate the interactions between TCP and ad hoc routing protocols. An initial study of the effect of routing on the quality of TCP communications is presented by Dyer and Boppana in [8]. Their study concentrates on TCP throughput that is achievable using different ad hoc routing schemes. Doing such measurements the authors mainly evaluate the quality of the particular routing scheme with respect to the path recovery times. The authors do not explicitly consider particular TCP-over-MANET problems.

The work of Perkins and Hughes 9 presents an evaluation of the effect of DSR and AODV on TCP performance. The authors find that when DSR is used as a routing protocol TCP achieves higher throughput than in the case of AODV.

In [10] Nahm et al. describe problems of interoperation between TCP and ondemand ad hoc routing protocols. They observe that TCP causes overreaction of the routing protocol, which degrades the quality of the end-to-end connection. The authors show cases where TCP, occupying the available bandwidth, prevents propagation of the control routing messages. This leads to large re-connect intervals, which in turn reduce the TCP throughput. The authors propose to use the 
known mechanisms for reducing TCP traffic load including an adaptive window increment schemes and delayed acknowledgments techniques.

The work in 11] from Uppsala University studies the interactions between TCP, UDP and routing protocols in MANETs. The authors show that the broadcast traffic generated by dynamic routing adds instability to ad hoc networking. In particular hidden terminal and channel capture effects cause instability of routes due to losses of control routing messages caused by the competing data traffic. This results in a very high packet loss rate for UDP traffic and long repeated timeouts of TCP.

\section{Problem Statement}

The performance of ad hoc routing protocols can be evaluated either with respect to the efficiency of establishing and maintaining the routing path between the communicating peers or with respect to the effect which routing traffic places on the quality characteristics of data flows. In both cases the process of the experimental performance evaluation is a complex task. In this paper we do not evaluate the considered routing protocols with respect to the quality of the path discovery phase. We concentrate on the second aspect of the routing performance and evaluate the degree of quality degradation for TCP connections due to routing activity.

\subsection{Current Performance Metrics and Why They Are Not Informative for Evaluating TCP+routing Interactions}

The major problem with the evaluation of TCP+routing interactions is the absence of TCP specific performance metrics for ad hoc routing protocols. In the literature related to the performance analysis of routing schemes [1, 8, 12, 13, we found very few quantitative performance metrics. The informational RFC 2051 [14 summarizes these metrics as follows:

1. Average number of control routing bits transmitted per data bit delivered, sometimes referred as to "Normalized routing load". This metric measures the overhead produced by transmission of routing messages.

2. Average number of data bits transmitted per data bit delivered, also referred as to "Packet delivery ratio". This metric is normally interpreted as a measure of the quality of data delivery within the network.

These two metrics are used mainly for two purposes. Firstly, to quantify a load produced by routing traffic and secondly, to assess the effect of routing on UDP based communications for which the packet loss rate is an illustrative performance characteristic. However, in the case of TCP communications, none of them is able to characterize the overall quality of the ongoing sessions.

As an example of why current performance metrics are not informative consider a scenario depicted in Fig. 1] and assume that the propagation of a routing message issued by Node $\mathrm{X}$ causes a loss of one data packet issued by Node $\mathrm{Y}$. 


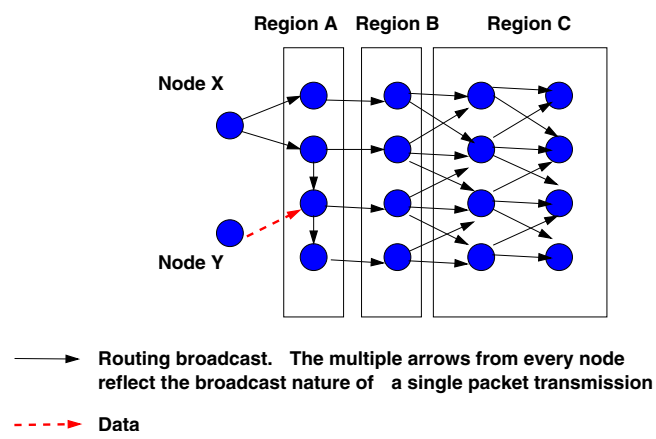

Fig. 1. Illustrative example of inability of the "normalized routing load" metric to capture the spatial effect of routing on data transmission

During the measurement period we observe 13 transmissions of routing messages in the entire network. The question is: How relevant is the information that 13 routing packets caused the loss of one data packet?

In fact only transmissions in Region B may cause the loss of our data packet if these transmission events coincide in time. If the transmission from Node Y happens at the same time as routing transmissions in Region A, Node Y will refrain from the transmission because it will sense the medium as busy. The nodes in Region $\mathrm{C}$ are located three hops away from Node $\mathrm{Y}$, therefore transmissions from this region would not interfere with our data packet. Finally, only transmissions in Region B will cause the loss of the data packet because of the hidden terminal effect. Therefore the actual effect of routing traffic on the data transmission in our example is four routing packets per one lost data packet. The major conclusion from this simple observation is that the "normalized routing load" metric does not reflect the spatial effect from the routing activity on the quality of data sessions.

As for the metric "Packet delivery ratio", it is indeed an informative measure of the routing activity but only with respect to UDP traffic. Evaluation of TCP performance is a more challenging task. The quality of TCP communications is characterized by a set of metrics including the throughput and the fairness metrics. In this respect knowing the proportion of the number of delivered TCP data segments to the number of emitted data segments, which would be captured by the "Packet delivery ratio" metric, is certainly not enough to assess the degree of service degradation for the particular TCP flow.

\subsection{Methodology Outline}

We see the assessment of the routing performance based on direct observation of transmission events for routing packets as problematic due to obvious difficulties of capturing the spatial effect of the routing traffic described above. We adopt the methodology behind the construction of the "Packet delivery ratio" metric and benchmark different routing schemes by monitoring the performance of TCP traffic. For this we first identify a set of TCP specific performance metrics. 
Our methodology is based on the definition of the "optimal" reference TCP performance. For this we use the results from [15], which describes an ingress throttling mechanism for achieving stable and predictable performance of the TCP protocol in multihop ad hoc networks with static routing.

In our simulation experiments we enable different routing schemes and monitor the deviation of the resulting TCP performance from the reference one. Up to a certain network scale the effect from transmissions of the control routing messages will be tolerable from the user's point of view. However, beyond that the routing traffic itself will cause a severe and unacceptable degradation of the TCP quality. We call the set of network configurations (in terms of the network size and the number of sustained TCP sessions) where routing traffic does not become a reason for poor TCP performance the admissible operation range (AOR) of MANETs.

Finally, at the border of the AOR, which we call the routing ad hoc horizon, we compute the "Equivalent routing load". This metric reflects the level of quality degradation for the data traffic caused by a specific ad hoc routing scheme.

\section{Admissible Operation Range, Routing Ad Hoc Horizon and Equivalent Routing Load}

\subsection{General Experimental Setup and TCP Performance Metrics}

For our simulation experiments we use the topology in Fig. 2(a). There we have three geographical areas with sources of TCP sessions, forwarder nodes and sink nodes. The forwarding area begins and ends one wireless hop away from the area with the sources and the sinks, respectively. The size of the forwarding area ensures three hops communications between each source and sink. The number of nodes in the forwarding area assures potential connectivity for each source-destination pair. In this topology we are able to vary two parameters: The number of competing TCP sessions and the number of nodes in the network. By increasing the number of simultaneously active sessions we increase the intensity of the on-demand routing traffic. By increasing the number of nodes in the forwarding region we increase the duration of the broadcast bursts, since more nodes are involved in the (re-) broadcasting process.

We used the ns-2 network simulator 3 (version 2.27) and TCP Newreno as the most popular variant of the protocol. We set the value of the TCP maximum segment size (MSS) to $600 \mathrm{~B}$. The data transmission rate of all devices is $2 \mathrm{Mb} / \mathrm{s}$; RTS/CTS handshake is disabled; Other ns-2.27 parameters have default values. In all simulations we used continuous FTP traffic from all sources.

Considered routing protocols. For the experiments we use AODV-UU [16], the stable and the RFC compliant implementation of AODV from Uppsala University. As well as LUNAR stable implementation which is available from [17.

3 [Online]. Available: http://www.isi.edu/nsnam/ns/ 
Two variants of AODV protocol are considered. In the first variant the HELLO mechanism is enabled to maintain the connectivity between neighbors further on we refer to this variant of AODV as to AODV-HELLO. In the second AODV variant, further referred as to AODV-LL, the route maintenance is done by means of explicit link layer feedback. In the former case the loss of connectivity between nodes forwarding traffic of a specific connection is detected by three missing HELLO messages; in this case the route maintenance procedure is invoked and the problem is signaled back to corresponding sources. In AODV-LL a packet loss during the transmission is detected by the link layer; the problem is then immediately reported to the routing engine, which in turn invokes the route maintenance operations.

The first variant of LUNAR uses standard settings as described in [7. The second variant has a modified periodic refresh timer as we describe later on in Sect. 4.3 .

Overall, the two variants of AODV and the two variants of LUNAR produce four distinct patterns of routing traffic in the networks. These patterns fit into two major types of the broadcast traffic invocation: (a) Error-driven (AODV-LL and opportunistic LUNAR) and (b) periodic (AODV-HELLO and LUNAR).

Used performance metrics. We assess TCP performance using the following set of metrics:

- Combined (total) TCP throughput of all existing in the network TCP flows. Denote this metric $T h r_{t o t}$;

- Unfairness index $u$ :

$$
u=1-\frac{\left(\sum_{i=1}^{N} T h r_{i}\right)^{2}}{N \sum_{i=1}^{N} T h r_{i}^{2}} .
$$

It is the opposite to the classic Jain fairness index, where $T h r_{i}$ is the throughput of FTP session $i$ and $N$ is the number of active sessions. A value of 0 for the unfairness index $u$ means that all TCP sessions receive the same share of the network's total capacity, while an unfairness of 1 means that only few sessions are monopolizing all bandwidth;

- The unsmoothness metric as defined in Appendix $\mathrm{A}$ for the qualitative assessment of the TCP session progress.

\subsection{Reference "Optimal" TCP Performance}

In order to obtain a stable and predictable behavior of the TCP protocol in multihop IEEE 802.11 based ad hoc network we use the ingress rate throttling scheme presented in [15]. There the max-min fairness framework from the wireline Internet is adapted to the specifics of MANETs and practically implementable mechanisms are proposed to enforce the model. The two major components of this solution are: (a) The usage of an ideal throughput achieved by a multihop TCP flow for characterizing the boundary load of a geographical region traversed by a multihop TCP session and (b) the rate throttling mechanism for reducing 


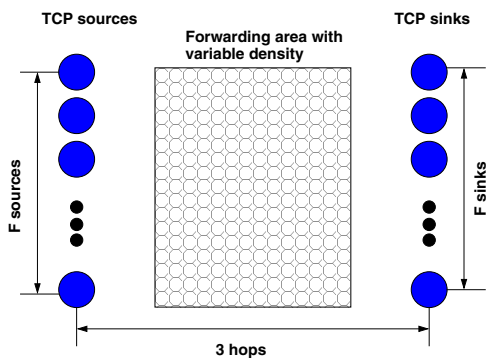

Net size $\mathrm{N}=$ sources+sinks+forwarders

(a) Experimental topology.

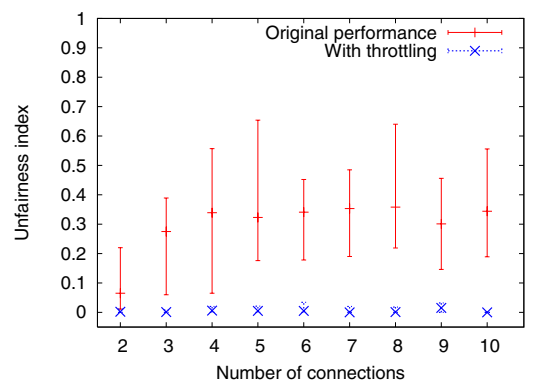

(c) Unfairness index vs. \# connections.

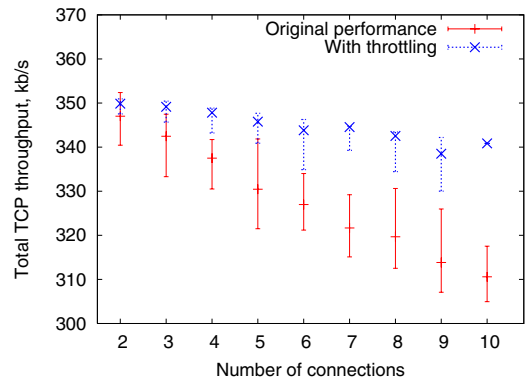

(b) Total TCP throughput vs. \# connections.

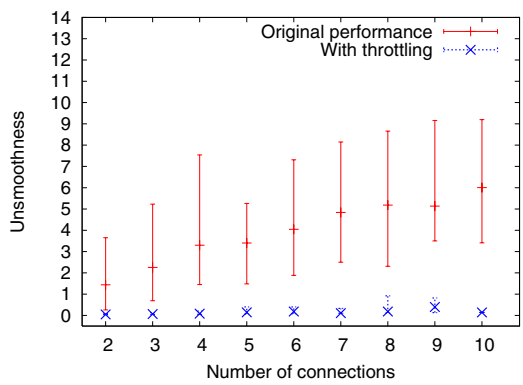

(d) Unsmoothness vs. \# connections.

Fig. 2. Experimental topology and reference "optimal" TCP performance in multihop ad hoc networks achieved with the ingress throttling scheme

the output rate at sources of TCP sessions in order to control the load in their bottleneck regions. The throttling level for each TCP session is given by (2).

$$
r_{i}^{\text {ingress }} \leq \frac{T h r_{\max }\left(h, M S S, T X_{802.11}\right)}{\rho_{\max }}
$$

In the above formula $T h r_{\max }\left(h, M S S, T X_{802.11}\right)$ is the maximal throughput of a TCP flow traversing $h$ hops, transmitting data segments of size $M S S$ while $T X_{802.11}$ is the used transmission rate at the physical layer along the path of the flow and $\rho_{\max }$ is the maximum number of competing TCP flows along the path of a specific session (path density). The value of the maximal TCP throughput for the particular set of parameters can be either formally or experimentally estimated and made available at the source nodes. The above transmission rate limit is used to set the delay parameter of the scheduler for the queue with locally generated packets at layer 2.5 at each source of TCP connections. The delay parameter is computed as $\Delta=\frac{M S S_{i}}{r_{i}^{\text {ingress }}\left(h, M S S, T X_{802.11}\right)}$.

The two major properties of ingress throttling [15], which we utilize for benchmarking the routing protocols, are: (a) the smooth progress of shaped TCP flows and (b) the optimal utilization of the network when all competing TCP flows 
are active. We illustrate these properties by an experiment as follows. We use the topology depicted in Fig. 2(a) The routes for all connections are statically assigned prior to the start of communications. We vary the number of connections in the network from 2 to 9 and activate them simultaneously; For each case we run 30 simulations with enabled and disabled ingress rate throttling.

The sub-figures 2(b) - 2(d) show the dynamics of the combined (total) TCP throughput in the network, the unfairness index and the unsmoothness metrics for different numbers of competing connections. All graphs show the mean values and the range between minimal and maximal values of the corresponding metrics over 30 simulation runs. In each run we seeded the random number generator of ns-2 randomly.

From sub-figure 2(b) we observe that performing the throttling actions, the resulting total throughput is equal to or larger than in the case without throttling. This demonstrates the increase in network utilization due to throttling. From sub-figures 2(c) and 2(d) we can see an improvement of the quality of TCP communications with respect to the unfairness and the unsmoothness metrics. When the ingress throttling is enabled all flows are smooth and free from long interruptions; overall almost perfect fairness is achieved.

It is important to point out the stability of the observed performance values. As it is visible from the graphs, the service offered by the plain combination of TCP and MAC 802.11 is highly variable and to a large extend unpredictable.

\subsection{Admissible Operation Ranges}

The stability and predictability of TCP performance demonstrated above is the needed reference behavior of the TCP protocol which we utilize for the analysis of the ad hoc routing schemes. When adding a routing protocol to the network with enabled ingress throttling scheme, the resulting performance will deviate from the observed "optimal" one. We predict that the uncontrolled broadcast transmissions that are present in all popular ad hoc routing schemes impose a limit on network size and number of sustained TCP sessions which we call the "admissible operation range".

Description of AOR experiments. In order to determine the "admissible operation range" we performed a series of simulation based experiments. In all experiments the ingress throttling is enabled. Each experiment is performed for the particular routing protocol with a fixed number of simultaneously active TCP sessions and a variable number of nodes in the forwarding area of the topology in Fig. 2(a).

We start with the minimum number of the forwarding nodes to ensure connectivity for every TCP connection (two nodes). We run simulations with this configuration up to 30 times and measure the worst unsmoothness amongst the competing flows and the unfairness index in each run. If after 30 runs the average of the worst unsmoothness is less than 1 and the worst unfairness index is less than $10 \%$ we increase the number of nodes in the forwarding area and repeat the experiment. We continue to increase the number of forwarding nodes until 
either of the metrics goes beyond the corresponding threshold. At this point we record the last network configuration (the number of TCP sessions and the network size) and the resulting combined (total) TCP throughput. After that we increase the number of simultaneously active TCP connections and repeate the sequence of experiments.

During the experiments with AODV-HELLO the best measured values of the unsmoothness metric is between one and two. For this variant of AODV we determine the "weak $A O R$ " as it is described below.

The results for the two variants of AODV protocol and for LUNAR are shown in Fig. 3. The shaded areas in the figures show the admissible operation range where the unsmoothness-unfairness property is acceptable for all competing TCP flows. Outside the shaded area either the value of the unsmoothness or the unfairness metric becomes unacceptable for at least one end user.

The left slopes of the AOR show the minimal network configurations where the particular number of distinct connections is possible. For example, four distinct three-hop flows are possible when we have one source node, four destinations and two forwarding nodes, that is in total 7 nodes in the network. The right border of the corresponding AORs represents larger network configurations for the particular number of TCP sessions. For example, in the case where AODV with link layer feedback is used, four connections with the acceptable unsmoothness and unfairness metrics can exist in a network with four distinct sources, four destinations and 22 forwarding nodes, that is 30 network nodes in total.

AODV-LL. As we observe from Fig. 3(a) the least impact on the ideal TCP behavior is introduced by the routing traffic pattern of AODV with link layer feedback enabled. This is because of the error-driven invocations of broadcast transmissions. In AODV-LL the broadcast activities are initiated as a reaction to packet losses reported by the link layer to the AODV engine. In the case of a packet loss the protocol assumes that the connectivity to the corresponding neighbor is lost and initiates the route recovery procedure. When the ingress throttling scheme is enabled, the collisions between data packets are not frequent (this is indicated by the close to zero unsmoothness metric in Fig. 2(d)]. In this case the reason for a higher packet loss rate, hence more frequent invocation of the path recovery phase, is the routing activity itself. As we observe in networks of up to 30 nodes, the broadcast traffic does not introduce enough overhead to force TCP flows into the routing induced slow start phase. However, beyond 30 nodes the broadcast bursts caused by every lost packet are long enough to cause the invocation of the slow start phase at a TCP sender; this leads to stammering TCP flow progress and worse fairness figures.

AODV-HELLO. The most unstable effect on the quality of TCP sessions is introduced by the traffic patterns produced by AODV-HELLO. In all experiments the unsmoothness of the competing TCP flows was between one and two at best. The analysis of the communication traces reveals that this is due to transmissions of HELLO messages. Despite of the small size, their frequent and independent emissions from multiple nodes does not allow any of the competing 


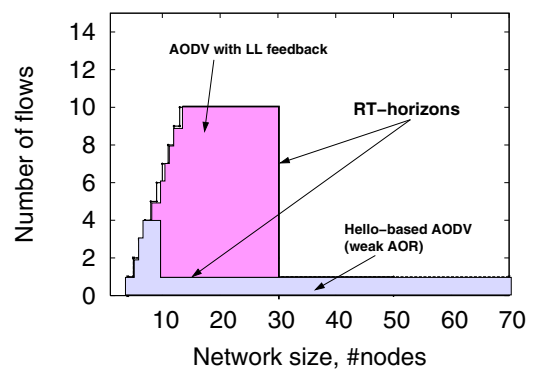

(a) AODV-LL and AODV-HELLO.

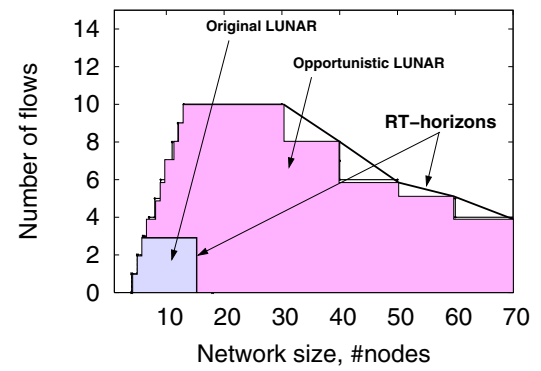

(b) Original and opportunistic LUNAR.

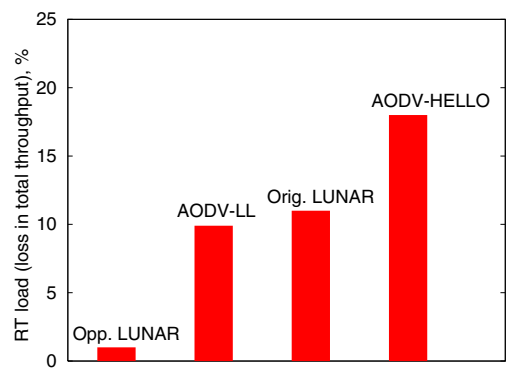

(c) Equivalent routing load.

Fig. 3. Admissible operation ranges and equivalent routing load for the considered routing protocols

TCP sessions to progress smoothly through the network. Even though we could not observe perfectly smooth flows as we did in the case of AODV-LL, we decided to delimit the admissible operation range where $0<$ Unsmoothness $<2$ and the unfairness index (1) is less than 10\%. We labeled this admissible operation range for AODV-HELLO as "weak AOR" in Fig. 3(a).

LUNAR. As for the effect of the routing traffic pattern generated by LUNAR, we observe that it is very similar to that of AODV-HELLO. The smaller shaded area in Fig. 3(b) shows the admissible operation range for the original LUNAR with its forced three seconds complete route re-discoveries. The difference is that the horizon with LUNAR is stable, meaning that the unsmoothness metric for all flows within the AOR is equal to or less than 1 and the fairness is close to perfect. The stability of LUNAR's admissible operation range can be explained by less frequent initiations of the broadcast traffic than in the case of AODVHELLO. Certain similarities in the right border of the AOR are explained by the fact that LUNAR's three second forced route rediscovery interval is chosen with the reference to the HELLO interval in AODV: It corresponds to two HELLO rounds which are needed by AODV to determine a change of the route. However, in the case of LUNAR we have a strict right border. This can be explained by the difference in the nature of broadcast patterns generated by the two protocols. 
If in the case of AODV-HELLO we have short frequent one hop broadcasts, in the case of LUNAR we have less frequent but more massive flooding waves, which result in stammering TCP progress with increasing number of nodes in the network.

Opportunistic LUNAR. As can be seen in Fig. 3(b) the original LUNAR has a narrow admissible operation range. As an experiment we changed the route refresh strategy of LUNAR to one which closely resembles the error-driven pattern of AODV-LL. To do this we disabled the forced three second complete route rediscovery mechanism. Instead, we retain a route as long as there are packets arriving to the forwarding engine: With every new data packet we shift the route timeout three seconds into the future. This modification allowed us to create an "opportunistic" version of LUNAR.

This change allowed us to significantly extend the horizon. Moreover, we achieved even better characteristics in comparison to AODV-LL. Now the right edge of the admissible operation range stretches to bigger networks. This is because we do not interpret every loss of a packet as an indication of the route breakage as it is the case with AODV-LL. Instead, we react on invocations of slow starts in TCP which are less frequent events, given that the ingress throttling scheme is deployed in all sources. By doing so we significantly decrease the frequency of broadcast bursts.

\subsection{Routing Ad Hoc Horizon and Equivalent Routing Load}

In Sect. 3 we presented the reasoning for why the estimation of the routing load is complex and not an obvious task. Recapitulating, this is due to the difficulties to capture the spatial effect of the broadcast traffic on a particular data session. Because of this the averaged metrics for routing load do not reflect the spatially distinct effects of the routing traffic on the ongoing data sessions. In the previous subsection we described a way to quantify the admissible operation region for reactive ad hoc routing protocols and TCP communications. The routing ad hoc horizon represents the critical network size where TCP flows maximally use the network capacity which is not consumed by transmission of routing messages and have an acceptable end user quality. Beyond this horizon, TCP flows begin to suffer from routing induced unfairness. In this case the progress of affected flows becomes disrupted, potentially leading to long disconnection intervals and loss of network utilization. The important property of the stability of communications within the AOR is that we can indirectly estimate the routing load produced by one or another protocol.

We define an "equivalent routing load" metric as follows. Assuming that our ingress throttling is deployed in all sources and that we measure the total TCP throughput in the network without routing $\left(T h r_{\text {norouting }}^{\text {tot }}\right)$, in the case where all competing flows are active the network is fully utilized. Now we measure the total TCP throughput on the right border of the admissible operation range $\left(T h r_{\text {withrouting }}^{\text {tot }}\right)$. In this case the competing TCP flows maximally use the capacity that is left from the routing traffic. Then the quantity $\left(1-\frac{T h r_{\text {withrouting }}^{\text {tot }}}{T h r_{\text {norouting }}^{\text {tot }}}\right) \cdot 100 \%$ 
reveals the reduction in the throughput in per-cent 4 due to the routing activity. Figure 3(c) shows the equivalent routing load for the considered protocols.

We may observe that in addition to the very narrow admissible operation range the HELLO-based AODV consumes more useful data throughput than other protocols. As for the modified opportunistic LUNAR protocol, its "equivalent routing load" is the smallest among all routing protocols. This is because the routing activity in this case happens only during the path establishment of each flow. After that the ingress throttling scheme prevents invocation of slow starts in the stable network as we have in our experiments. Therefore no further routing traffic is involved after all sessions were successfully established until the end of simulations.

\subsection{Summary}

In this section we developed our approach for the analysis of the effect of ad hoc routing traffic on the quality of TCP communications. For each of the the two considered routing protocols and static topologies we determined the admissible operation range, which is the set of network configurations where routing and TCP traffic can peacefully co-exist.

We found that 10 - 20 nodes is a critical network size for routing schemes which utilize periodic broadcast invocations in their route maintenance phase. With such protocols only few TCP connections may exist with an acceptable quality. It is worth noting that this type of broadcast invocations is present in the specification of a new generation reactive routing protocol for MANETs. In the recent draft for DyMo [18 the HELLO mechanism for the connectivity maintenance between the neighbors is inherited from AODV. In contrast, our analysis shows that a "TCP-friendly" ad hoc routing protocols should use mainly an error-driven form of broadcast invocations.

\section{Conclusions}

In this paper we analyzed the effect of different routing traffic patterns on the performance of TCP during stable operations of MANETs. We found that quantifying the routing load is difficult even in static networks without mobility. We highlighted the fact that the current performance metrics for the evaluation of routing protocols are not informative enough for the evaluation of $\mathrm{TCP}+$ routing interactions.

We suggested a methodology for an indirect measurement of the routing load and quantification of the routing effect on TCP performance. Using this technique we were able to show that the routing traffic itself can be a reason for poor TCP performance in MANETs.

We analyzed two routing protocols with four distinct routing traffic patterns and were able to identify the scaling region of MANETs (in terms of the number

\footnotetext{
${ }^{4}$ We intentionally do not express the "Equivalent routing load" in bits per second since this value would be specific to the particular transmission speed picked in the experimental environment (in our case ns-2).
} 
of simultaneously active TCP sessions and the number of network nodes) beyond which the traffic generated by the routing protocol significantly degrades the performance of TCP. We call this region the "admissible operation range" of MANETs.

Our major conclusion regarding the effect of routing traffic patterns on TCP communications is that periodic, non error-driven broadcasts of even short messages is harmful for data communications and leads to narrowing the operational region of MANETs.

Further studies have to add mobility to the scene, which we expect to become another limiting factor for the admissible operation range.

\section{References}

1. J. Broch, D. Maltz, D. Johnson, Y-C.Hu, and J. Jetcheva, "A performance comparison of multi-hop wireless ad hoc network routing protocols," in Proc. ACM MobiCom'98, Dallas, TX, USA, 1998.

2. K. Xu, S. Bae, S. Lee, and M. Gerla, "TCP behavior across multi-hop wireless networks and the wired Internet," in Proc. WoWMoM'02, Atlanta, GA, USA, Sep. 2002 .

3. H. Balakrishnan, V. Padmanabhan, S.Seshan, and R. H. Katz, "A comparison of mechanisms for improving TCP performance over wireless links," in Proc. ACM SIGCOMM'05 workshops, Philadelphia, PA, USA, Aug. 2005.

4. H. Elaarag, "Improving TCP performance over mobile networks," ACM Computing Surveys, vol. 34, no. 3, Sept. 2003.

5. S. Xu and T. Saadawi, "Does the IEEE 802.11 MAC protocol work well in multihop wireless ad hoc networks?" IEEE Communications Magazine, Jun. 2001.

6. C. Perkins, E. Belding-Royer, and S. Das, "Ad hoc on-demand distance vector (AODV) routing," IETF RFC 3561, Jul. 2003. [Online]. Available: http://www.rfc-editor.org/rfcsearch.html

7. C. Tschudin, R. Gold, O. Rensfelt, and O. Wibling, "LUNAR: a lightweight underlay network ad-hoc routing protocol and implementation," in Proc. NEW2AN'04, St. Petersburg, Russia, Feb. 2004.

8. T. Dyer and R. Boppana, "A comparison of TCP performance over three routing protocols for mobile ad hoc networks," in Proc. ACM MobiHoc'01, Long Beach, CA, USA, Oct. 2001.

9. D. Perkins and H. Hughes, "Investigating the performance of TCP in mobile ad hoc networks," International Journal of Computer Communications, vol. 25, no. 11-12, 2002.

10. K. Nahm, A. Helmy, and C.-C. J. Kuo, "TCP over multihop 802.11 networks: issues and performance enhancement," in Proc. ACM MobiHoc'05, Urbana-Campaign, Illinoise, USA, May 2005.

11. C. Rohner, E. Nordström, P. Gunningberg, and C. Tschudin, "Interactions between TCP, UDP and routing protocols in wireless multi-hop ad hoc netwrorks," in Proc. IEEE ICPS Workshop on Multi-hop Ad hoc Networks: from theory to reality (REALMAN'05), Santorini, Greece, Jul. 2005.

12. S.-J. Lee, E. Belding-Royer, and C. Perkins, "Scalability study of the ad hoc ondemand distance vector routing protocol," International Journal of Network Management, vol. 13, no. 2, Mar./Apr. 2003. 
13. S. R. Das, C. Perkins, and E. M. Royer, "Perfortmance comparison of two ondemand routing protocols for ad hoc networks," in Proc. IEEE Infocom'00, TelAviv, Israel, Mar. 2000.

14. S. Corson and J. Macker, "Mobile ad hoc networking (MANET): Routing protocol performance issues and evaluation considerations," IETF Infromational RFC 2501, 1999. [Online]. Available: http://www.rfc-editor.org/rfcsearch.html

15. E. Osipov, "On the practical feasibility of fair TCP communications in IEEE 802.11 based multihop ad hoc wireless networks," Ph.D. dissertation, University of Basel, Switzerland, 2005.

16. AODV-UU implementation, Uppsala University. [Online]. Available: http:// core.it.uu.se/AdHoc/AodvUUImpl

17. Uppsala University ad hoc implementation portal. [Online]. Available: http:// core.it.uu.se/AdHoc/ImplementationPortal

18. I. Chakeres, E. Belding-Royer, and C. Perkins, "Dynamic MANET on-demand (DYMO) routing," IETF draft (work in progress), 2005. [Online]. Available: http://www.ietf.org/internet-drafts/draft-ietf-manet-dymo-02.txt

19. Ch.Tschudin and E. Osipov, "Estimating the ad hoc horizon for TCP over IEEE 802.11 networks," in Proc.MedHoc'04, Bodrum, Turkey, Jun. 2004.

\section{A Definition of the "unsmoothness" Metric}

As it is shown in [19] it is important to account for interruptions in TCP flow progress since some of the flows may suffer from long no-progress intervals although the throughput and fairness metrics would report positive network performance.

In order to qualitatively assess the progress of each TCP session we construct an "unsmoothness" metric as is illustrated in Fig. 4. The construction of the metric is done during the analysis of communication traces. After the end of each test run we parse the packet trace and record the progress of the received sequence numbers in time for each flow and the corresponding start times for every session. The stop time for all sessions is assumed to be the same. With this

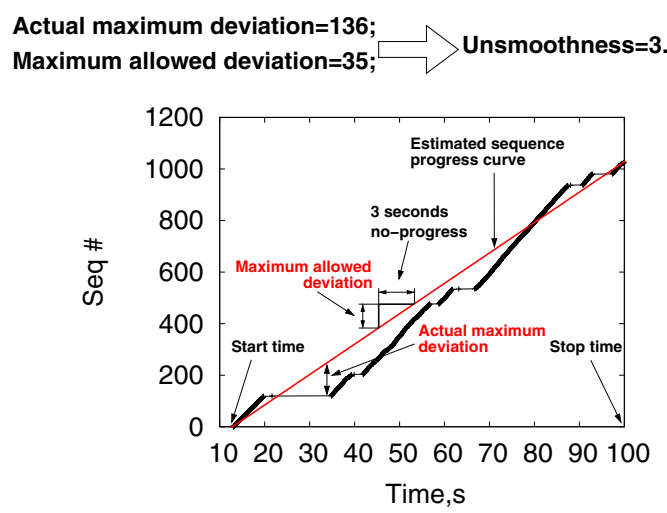

Fig. 4. Computation of the unsmoothness metric 
information we estimate the ideal curve of the sequence number progress. It is shown by the straight line in Fig. 4. After that we analyze the recorded process of sequence number arrivals for each flow and compare each received sequence number with the estimated "smooth" value for the corresponding time. The result of the comparison is the absolute deviation of the actual sequence number from the estimated value. Obviously, in most of the measurements we will have some deviation from the estimated curve even for a perfectly smooth flow due to rounding errors. In order to allow some small deviations we compute the maximum allowed deviation. This value corresponds to the three seconds noprogress time which was chosen based on our empirical observations that longer no-progress times definitely indicate the presence of the TCP unfairness in ad hoc networks. Finally, we compute the "unsmoothness" metric as in (3).

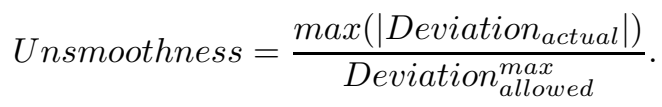

The "unsmoothness" metric is always larger than zero. We say that the quality of a TCP flow is acceptable for an end-user if Unsmoothness $\leq 1$. 